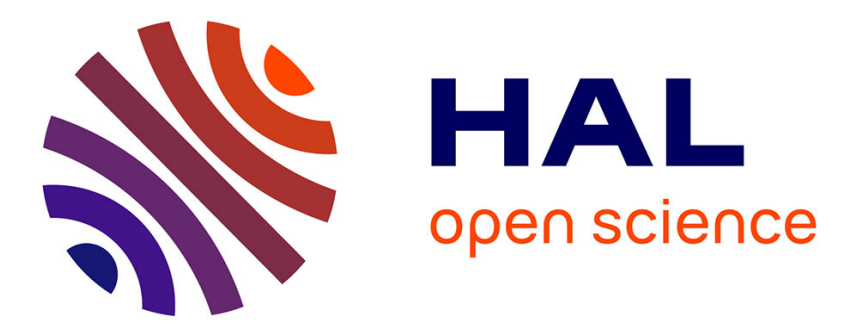

\title{
Indirect Adaptive Attenuation of Multiple Narrow-Band Disturbances Applied to Active Vibration Control
}

\author{
Tudor-Bogdan Airimitoaie, Ioan Doré Landau
}

\section{To cite this version:}

Tudor-Bogdan Airimitoaie, Ioan Doré Landau. Indirect Adaptive Attenuation of Multiple NarrowBand Disturbances Applied to Active Vibration Control. IEEE Transactions on Control Systems Technology, 2014, 22 (2), pp.761-769. 10.1109/TCST.2013.2257782 . hal-00830039

\section{HAL Id: hal-00830039 https://hal.science/hal-00830039}

Submitted on 14 Jun 2019

HAL is a multi-disciplinary open access archive for the deposit and dissemination of scientific research documents, whether they are published or not. The documents may come from teaching and research institutions in France or abroad, or from public or private research centers.
L'archive ouverte pluridisciplinaire HAL, est destinée au dépôt et à la diffusion de documents scientifiques de niveau recherche, publiés ou non, émanant des établissements d'enseignement et de recherche français ou étrangers, des laboratoires publics ou privés. 


\title{
Indirect Adaptive Attenuation of Multiple Narrow-band Disturbances Applied to Active Vibration Control
}

\author{
Tudor-Bogdan Airimiţoaie and Ioan Doré Landau
}

\begin{abstract}
In this paper, an indirect adaptive control methodology for attenuation of multiple unknown time varying narrowband disturbances is proposed. The method is based on the real time estimation of the frequency of narrow-band disturbances using adaptive notch filters (ANF) followed by the design of a controller using adjustable band-stop filters (BSF) for the appropriate shaping of the output sensitivity function. A YoulaKučera parametrization of the controller is used for reducing the computation load. This approach is compared on an active vibration control (AVC) system with the direct adaptive control scheme based on the internal model principle (IMP) proposed in [1]. Real time experimental results are provided.
\end{abstract}

Index Terms-Indirect adaptive regulation, active vibration control, loop-shaping, Youla-Kučera parametrization, multiple narrow-band disturbances.

\begin{tabular}{lll} 
& \multicolumn{1}{c}{ LIST OF ACRONYMS } \\
ANF & - Adaptive notch filter \\
AVC & - Active vibration control \\
BSF & - & Band-stop filter \\
FIR & - & Finite impulse response \\
IIR & - & Infinite impulse response \\
IMP & - & Internal model principle \\
PSD & - & Power spectral density
\end{tabular}

\section{INTRODUCTION}

A $\mathrm{N}$ important problem in active vibration (or noise) control is the compensation of disturbances without measuring them. In this case, a feedback approach is considered for disturbance attenuation. In general, one considers the disturbances as being a white noise or a Dirac impulse passed through a filter which characterizes the model of the disturbance. For the purpose of this paper, the disturbances are considered to be unknown and/or time varying multiple narrow-band disturbances, in other words their model has time varying coefficients ${ }^{1}$. This motivates the use of an adaptive regulation approach since the objective is the attenuation of unknown disturbances without measuring them.

A popular methodology for this adaptive regulation problem is the design of a controller that incorporates the model of the

The authors are with the Control System Department of GIPSA-lab, St. Martin d'Héres, 38402 FRANCE, email: [tudor-bogdan.airimitoaie, ioandore.landau]@gipsa-lab.grenoble-inp.fr.

Tudor-Bogdan Airimiţoaie is also with the Faculty of Automatic Control and Computers, University "Politehnica" of Bucharest, Bucharest, 060042 ROMANIA, email: tudor.airimitoaie@acse.pub.ro.

${ }^{1}$ Throughout the paper it is assumed that the number of multiple narrowband disturbances is known (it can be estimated from data if necessary) but their frequency characteristics are not known. disturbance (internal model principle). Using the Youla-Kučera parametrization of the controller a direct adaptation technique can be implemented. This technique has been described in [1], [2], [3], [4], [5], [6]. Using the IMP principle, the complete rejection of the disturbances is attempted (asymptotically). In the case of several narrow-band disturbances, using IMP it is very difficult to find a controller such that the "water bed" effect on the output sensitivity function (amplification introduced at the other frequencies than those of the disturbances) be acceptable in terms of performance and robustness (see also [7]).

In practice, however, we do not need a complete rejection of the narrow-band disturbances but just a level of attenuation. Introducing only a level of attenuation combined with an appropriate controller design will reduce the "water bed" effect on the output sensitivity function improving both robustness and performance (by reducing the unwanted amplification of the noise). This will become particularly useful in the case of multiple narrow-band disturbances.

In this paper, a procedure for achieving an imposed attenuation (and not complete cancellation) is proposed. The procedure is based on the shaping of the output sensitivity function using band stop-filters (BSF) centered at the frequencies corresponding to spikes in the spectrum of the disturbance. The peculiarity of this approach is that the zeros of these BSF are implemented in the controller while the poles of the BSF are introduced as desired poles of the closed loop (see also [8], [9]). In this paper, the design of the BSF for narrow-band disturbance attenuation is further simplified by considering a Youla-Kučera parametrization of the controller ([4], [10], [11], [12]). By doing this, the dimension of the matrix equation that has to be solved is reduced significantly and therefore the computation load will be much lower. This is very important in the perspective of using this controller design procedure in an adaptive scheme.

In order to implement this approach in the presence of unknown narrow-band disturbances, one needs to estimate in real time the frequencies of the spikes contained in the disturbance. System identification techniques can be used to estimate the ARMA model of the disturbance ([1], [13]). Unfortunately, to find the frequencies of the spikes from the estimated model of the disturbance requires computation in real time of the roots of an equation of order $2 \cdot n$, where $n$ is the number of spikes. Therefore this approach is applicable in the case of one eventually two narrow-band disturbances. What is needed is an algorithm which can directly estimate the frequencies 
of the various spikes of the disturbance. Several methods have been proposed by the signal processing community for solving this issue ([14]). From these, the adaptive notch filter (ANF) is particularly interesting and has been reviewed in a number of articles ([15], [16], [17], [18], [19], [20], [21]). In this paper, the estimation approach presented in [22], [23] will be used. Combining the frequency estimation procedure and the control design procedure, an indirect adaptive regulation system for attenuation of multiple unknown and/or time varying narrowband disturbances is obtained.

In the present context, the hypothesis of constant dynamic characteristics of the AVC system is made (like in [1]). Furthermore, the corresponding control model is supposed to be accurately identified from input/output data.

In [24], a review of available methods for the rejection of narrow-band disturbances is given. In [4], the direct adaptive regulation of narrow-band disturbances using IMP and the Youla-Kučera parametrization is described and analyzed and extended in [1] for multiple disturbances. Another method for narrow-band disturbances rejection by feedback is based on the use of a disturbance observer ([25], [26], [27]). In [27], the disturbance observer is combined with a modified IMP scheme that uses an IIR filter, which acts as a frequency selector, to minimize the effect of the adaptive control on the output sensitivity function.

An indirect method that combines the frequency estimator of [17] with feedforward rejection of disturbances is presented in [28]. The method is also compared to a direct algorithm based on the phase-locked loop structure considered in communication systems.

This paper is organized as follows. In Section II, the main notations and equations for the indirect adaptive system are given. The controller design based on the use of BSFs is presented in Section III. A reduced complexity implementation of this method using the Youla-Kučera parametrization is then given in Section IV. The estimation method used for tracking the variations of the disturbances' frequencies is briefly described in Section V. The stability analysis of the proposed method is given in Section VI. In Section VII, an experimental performance evaluation of the resulting indirect adaptive regulation scheme and a comparison with the direct regulation method using Youla-Kučera parametrization of the controller and IMP [1] are presented. Some concluding remarks are given in Section VIII.

\section{SySTEM DESCRIPTION}

The basic indirect adaptive control bloc diagram used is shown in fig. 1 . The process output can be written $a^{2}$

$$
y(t)=G\left(q^{-1}\right) \cdot u(t)+p(t)
$$

where

$$
G\left(q^{-1}\right)=q^{-d} \frac{B\left(q^{-1}\right)}{A\left(q^{-1}\right)}
$$

\footnotetext{
${ }^{2}$ The complex variable $z^{-1}$ will be used to characterize the system's behavior in the frequency domain and the delay operator $q^{-1}$ will be used for the time domain analysis.
}

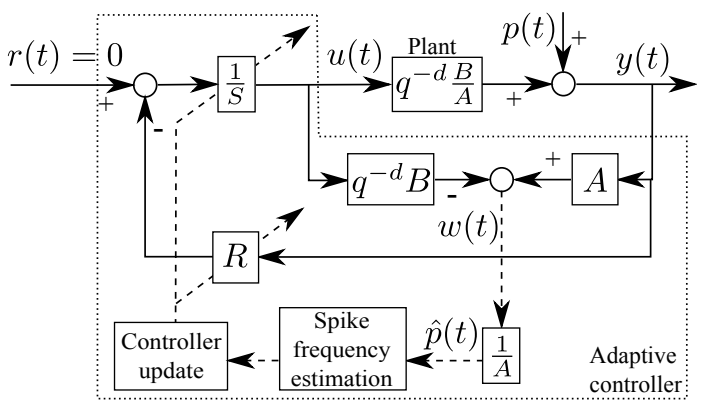

Fig. 1. Basic schema for indirect adaptive control.

is called the secondary path of the system and

$$
p(t)=\frac{D\left(\rho q^{-1}\right)}{D\left(q^{-1}\right)} \delta(t), \quad \rho \in(0,1) \text { is a fixed constant }
$$

is the effect of the disturbance on the measured output ${ }^{3}$.

As specified in the introduction, the hypothesis of constant dynamic characteristics of the AVC system is considered (similar to [1], [4]). The denominator of the secondary path model is given by

$$
A\left(q^{-1}\right)=1+a_{1} q^{-1}+\ldots+a_{n_{A}} q^{-n_{A}},
$$

the numerator is given by

$$
B\left(q^{-1}\right)=b_{1} q^{-1}+\ldots+b_{n_{B}} q^{-n_{B}}=1+q^{-1} B^{*}\left(q^{-1}\right)
$$

and $d$ is the integer delay (number of sampling periods) ${ }^{4}$.

The control signal is given by

$$
u(t)=-R\left(q^{-1}\right) \cdot y(t)-S^{*}\left(q^{-1}\right) \cdot u(t-1),
$$

with

$$
\begin{aligned}
S\left(q^{-1}\right) & =1+q^{-1} S^{*}\left(q^{-1}\right)=1+s_{1} q^{-1}+\ldots+s_{n_{S}} q^{-n_{S}} \\
& =S^{\prime}\left(q^{-1}\right) \cdot H_{S}\left(q^{-1}\right), \\
R\left(q^{-1}\right) & =r_{0}+r_{1} q^{-1}+\ldots+r_{n_{R}} q^{-n_{R}} \\
& =R^{\prime}\left(q^{-1}\right) \cdot H_{R}\left(q^{-1}\right),
\end{aligned}
$$

where $H_{S}\left(q^{-1}\right)$ and $H_{R}\left(q^{-1}\right)$ represent fixed (imposed) parts in the controller and $S^{\prime}\left(q^{-1}\right)$ and $R^{\prime}\left(q^{-1}\right)$ are computed. Under the hypothesis that the plant model parameters are constant and that an accurate identification experiment can be run, a reliable estimate $\hat{p}(t)$ of the disturbance signal can be obtained by using the following disturbance observer

$$
\hat{p}(t+1)=y(t+1)-q^{-d} \frac{B^{*}\left(q^{-1}\right)}{A\left(q^{-1}\right)} u(t),
$$

as shown in fig. 1. The disturbance estimator $(\hat{p}(t))$ is followed by a block which estimates spikes' frequencies and computes in real time the controller parameters.

\footnotetext{
${ }^{3}$ The disturbance passes through a so called "primary path" which is not represented in this figure, and $p(t)$ is its output

${ }^{4}$ As indicated earlier, it is assumed that a reliable model identification is achieved and therefore the estimated model is assumed to be equal to the true model.
} 


\section{A Controller Design Procedure for ATTENUATION OF MULTIPLE NARROW-BAND DISTURBANCES}

The purpose of this method is to allow the possibility of choosing the desired attenuation and bandwidth of attenuation for each of the estimated narrow-band disturbances. Choosing the level of attenuation and the bandwidth allows to preserve acceptable characteristics of the sensitivity functions outside the attenuation bands and this is very useful in the case of multiple narrow-band disturbances. This is the main advantage with respect to classical internal model methods which in the case of several narrow-band disturbances, as a consequence of complete cancellation of the disturbances, may lead to unacceptable values of the modulus of the output sensitivity function outside the attenuation regions. The controller design technique uses the shaping of the output sensitivity function for imposing the desired attenuation of narrow-band disturbances. It will be used for computing the parameters of the adjustable controller. The controller's parameters computation procedure will be presented considering, temporarily, constant and known frequencies of the narrow-band disturbances. The design uses BSFs to shape the output sensitivity functions. Following [8], [9], there exists a digital filter $\frac{S_{B S F_{i}}\left(z^{-1}\right)}{P_{B S F_{i}}\left(z^{-1}\right)}$ with the numerator included in the controller and the denominator as a factor of the desired closed-loop characteristic polynomial, which will assure the desired attenuation of a narrow-band disturbance (index $i \in\{1, \ldots, n\}$ ).

The BSFs have the following structure

$$
\frac{S_{B S F_{i}}\left(z^{-1}\right)}{P_{B S F_{i}}\left(z^{-1}\right)}=\frac{1+\beta_{1}^{i} z^{-1}+\beta_{2}^{i} z^{-2}}{1+\alpha_{1}^{i} z^{-1}+\alpha_{2}^{i} z^{-2}}
$$

resulting from the discretization of a continuous filter (see also [8], [9])

$$
F_{i}(s)=\frac{s^{2}+2 \zeta_{n_{i}} \omega_{i} s+\omega_{i}^{2}}{s^{2}+2 \zeta_{d_{i}} \omega_{i} s+\omega_{i}^{2}}
$$

using the bilinear transformation. This filter introduces an attenuation of

$$
M_{i}=-20 \cdot \log _{10}\left(\frac{\zeta_{n_{i}}}{\zeta_{d_{i}}}\right)
$$

at the frequency $\omega_{i}$. Positive values of $M_{i}$ denote attenuations $\left(\zeta_{n_{i}}<\zeta_{d_{i}}\right)$ and negative values denote amplifications $\left(\zeta_{n_{i}}>\right.$ $\left.\zeta_{d_{i}}\right)^{5}$.

Remark: The design parameters for each BSF are the desired attenuation $\left(M_{i}\right)$, the central frequency of the filter $\left(\omega_{i}\right)$ and the damping of the denominator $\left(\zeta_{d_{i}}\right)$. The denominator damping is used to adjust the frequency bandwidth of the BSF. For very small values of the frequency bandwidth the influence of the filters on frequencies other than those defined by $\omega_{i}$ is negligible. Therefore, the number of BSFs and subsequently that of the narrow-band disturbances that can be compensated can be large.

For $n$ narrow-band disturbances, $n$ BSFs will be used

$$
H_{B S F}\left(z^{-1}\right)=\frac{S_{B S F}\left(z^{-1}\right)}{P_{B S F}\left(z^{-1}\right)}=\frac{\prod_{i=1}^{n} S_{B S F_{i}}\left(z^{-1}\right)}{\prod_{i=1}^{n} P_{B S F_{i}}\left(z^{-1}\right)}
$$

${ }^{5}$ For frequencies bellow $0.17 f_{S}$ ( $f_{S}$ is the sampling frequency) the design can be done with a very good precision directly in discrete time ([8]).
As stated before, the objective is that of shaping the output sensitivity function. $S\left(z^{-1}\right)$ and $R\left(z^{-1}\right)$ are obtained as solutions of the Bezout equation

$$
P\left(z^{-1}\right)=A\left(z^{-1}\right) S\left(z^{-1}\right)+z^{-d} B\left(z^{-1}\right) R\left(z^{-1}\right),
$$

where

$S\left(z^{-1}\right)=H_{S}\left(z^{-1}\right) S^{\prime}\left(z^{-1}\right), \quad R\left(z^{-1}\right)=H_{R_{1}}\left(z^{-1}\right) R^{\prime}\left(z^{-1}\right)$, and $P\left(z^{-1}\right)$ is given by

$$
P\left(z^{-1}\right)=P_{0}\left(z^{-1}\right) P_{B S F}\left(z^{-1}\right) .
$$

In the last equation, $P_{B S F}$ is the product of the denominators of all the BSFs, (13), and $P_{0}$ defines imposed poles of the closed loop system in the absence of the disturbances (allowing also to satisfy robustness conditions). The fixed part of the controller denominator $H_{S}$ is in turn factorized into

$$
H_{S}\left(z^{-1}\right)=S_{B S F}\left(z^{-1}\right) H_{S_{1}}\left(z^{-1}\right),
$$

where $S_{B S F}$ is the combined numerator of the BSFs, (13), and $H_{S_{1}}$ can be used if necessary to satisfy other control specifications (in Section VII it is equal to 1 ). $H_{R_{1}}$ is similar to $H_{S_{1}}$ allowing to introduce fixed parts in the controller's numerator if needed (like opening the loop at certain frequencies). It is easy to see that the output sensitivity function becomes

$$
S_{y p}\left(z^{-1}\right)=\frac{A\left(z^{-1}\right) S^{\prime}\left(z^{-1}\right) H_{S_{1}}\left(z^{-1}\right) S_{B S F}\left(z^{-1}\right)}{P_{0}\left(z^{-1}\right) P_{B S F}\left(z^{-1}\right)}
$$

and the shaping effect of the BSFs upon the sensitivity functions is obvious. The unknowns $S^{\prime}$ and $R^{\prime}$ are solutions of

$$
\begin{gathered}
P\left(z^{-1}\right)=P_{0}\left(z^{-1}\right) P_{B S F}\left(z^{-1}\right)=A\left(z^{-1}\right) H_{S}\left(z^{-1}\right) S^{\prime}\left(z^{-1}\right)+ \\
+z^{-d} B\left(z^{-1}\right) H_{R_{1}}\left(z^{-1}\right) R^{\prime}\left(z^{-1}\right) .
\end{gathered}
$$

and can be computed by putting (18) into matrix form (see also [8]). The size of the matrix equation that needs to be solved is given by

$$
n_{B e z}=n_{A}+n_{B}+d+n_{H_{S_{1}}}+n_{H_{R_{1}}}+2 \cdot n-1,
$$

where $n_{A}, n_{B}$, and $d$ are respectively the order of the plant's model denominator, numerator, and delay (given in (4) and (5)), $n_{H_{S_{1}}}$ and $n_{H_{R_{1}}}$ are the orders of $H_{S_{1}}\left(z^{-1}\right)$ and $H_{R_{1}}\left(z^{-1}\right)$ respectively and $n$ is the number of narrow-band disturbances. Eq. (18) has an unique minimal degree solution for $S^{\prime}$ and $R^{\prime}$, if $n_{P} \leq n_{B e z}$, where $n_{P}$ is the order of the pre-specified characteristic polynomial $P\left(q^{-1}\right)$. Also, it can be seen from (18) and (16) that the minimal orders of $S^{\prime}$ and $R^{\prime}$ will be:

$$
n_{S^{\prime}}=n_{B}+d+n_{H_{R_{1}}}-1, \quad n_{R^{\prime}}=n_{A}+n_{H_{S_{1}}}+2 \cdot n-1 .
$$

Note that in an indirect adaptive regulation scheme, the Diophantine equation (18) has to be solved either at each sampling time (adaptive operation) or each time when a change in the narrow-band disturbances' frequencies occurs (self-tuning operation). 


\section{REducing the Computational LoAD OF the DESIGN BY USING THE YOULA-KUČERA PARAMETRIZATION}

The computational complexity related to the Bezout equation (18) is significant (in the perspective of its use in adaptive regulation). In this section, we show how the computation load of the design procedure can be reduced by the use of the YoulaKučera parametrization.

As before, a multiple band-stop filter, (13), should be computed based on the frequencies of the multiple narrowband disturbance. In what follows it will be shown that using a Youla-Kučera parametrization of the controller [12] a significant reduction of the computational load will be obtained.

Suppose that a nominal controller

$$
\begin{aligned}
R_{0}\left(z^{-1}\right) & =H_{R_{1}}\left(z^{-1}\right) R^{\prime \prime}\left(z^{-1}\right), \\
S_{0}\left(z^{-1}\right) & =H_{S_{1}}\left(z^{-1}\right) S^{\prime \prime}\left(z^{-1}\right)
\end{aligned}
$$

that assures nominal performances for the closed loop system in the absence of narrow-band disturbances is available. This controller satisfies the Bezout equation

$$
P_{0}\left(z^{-1}\right)=A\left(z^{-1}\right) S_{0}\left(z^{-1}\right)+q^{-z} B\left(z^{-1}\right) R_{0}\left(z^{-1}\right) \text {. }
$$

Since $P_{B S F}\left(z^{-1}\right)$ will define part of the desired closed loop poles, it is reasonable to consider a Youla-Kučera filter of the form $\frac{Q\left(z^{-1}\right)}{P_{B S F}\left(z^{-1}\right)}$ (which will automatically introduce $P_{B S F}\left(z^{-1}\right)$ as part of the closed loop poles). For this purpose, the controller polynomials are factorized as

$$
\begin{aligned}
R\left(z^{-1}\right)= & R_{0}\left(z^{-1}\right) P_{B S F}\left(z^{-1}\right)+ \\
& +A\left(z^{-1}\right) H_{R_{1}}\left(z^{-1}\right) H_{S_{1}}\left(z^{-1}\right) Q\left(z^{-1}\right), \\
S\left(z^{-1}\right)= & S_{0}\left(z^{-1}\right) P_{B S F}\left(z^{-1}\right)- \\
& -z^{-d} B\left(z^{-1}\right) H_{R_{1}}\left(z^{-1}\right) H_{S_{1}}\left(z^{-1}\right) Q\left(z^{-1}\right),
\end{aligned}
$$

where $Q\left(z^{-1}\right)$ is a FIR filter computed in order to satisfy (14) for $P\left(z^{-1}\right)=P_{0}\left(z^{-1}\right) P_{B S F}\left(z^{-1}\right)$, and $R_{0}\left(z^{-1}\right), S_{0}\left(z^{-1}\right)$ are given by (20) and (21) respectively. It can be seen from (23) and (24) that the new controller polynomials conserve the fixed parts of the nominal controller.

Taking into account (14), (16), (17), and (18), it remains to compute $Q\left(z^{-1}\right)$ such that

$$
S\left(z^{-1}\right)=S_{B S F}\left(z^{-1}\right) H_{S_{1}}\left(z^{-1}\right) S^{\prime}\left(z^{-1}\right) .
$$

Turning back to eq. (24) one obtains ${ }^{6}$

$$
S_{0} P_{B S F}=S_{B S F} H_{S_{1}} S^{\prime}+z^{-d} B H_{R_{1}} H_{S_{1}} Q .
$$

and taking into consideration also (21) it results

$$
S^{\prime \prime} P_{B S F}=S_{B S F} S^{\prime}+q^{-d} B H_{R_{1}} Q .
$$

In the last equation, the left side of the equal sign is known and on its right side only $S^{\prime}\left(z^{-1}\right)$ and $Q\left(z^{-1}\right)$ are unknown. This is also a Bezout equation which can be solved by finding the solution to a matrix equation of dimension

$$
n_{B e z_{Y K}}=n_{B}+d+n_{H_{R_{1}}}+2 \cdot n-1 .
$$

\footnotetext{
${ }^{6}$ The argument $\left(z^{-1}\right)$ has been dropped to simplify the writing of the equation.
}

As it can be observed, the size of the new Bezout equation is reduced in comparison to (19) by $n_{A}+n_{H_{S_{1}}}$. For systems with large dimensions, this has a significant influence on the computation time (in Section VII, $n_{A}=14$ and $n_{H_{S_{1}}}=0$ ). Taking into account that the nominal controller is an unique and minimal degree solution of the Bezout equation (22), we find that the left hand side of (27) is a polynomial of degree

$$
n_{S^{\prime \prime}}+2 \cdot n=2 \cdot n+n_{B}+d+n_{H_{R_{1}}}-1,
$$

which is equal to the quantity given in (28). Therefore, the solution of the simplified Bezout equation (27) is unique and of minimal degree. Furthermore, the order of the $Q$ FIR filter is equal to $2 \cdot n-1$.

Fig. 2 summarizes the implementation of the Youla-Kučera parametrized indirect adaptive controller.

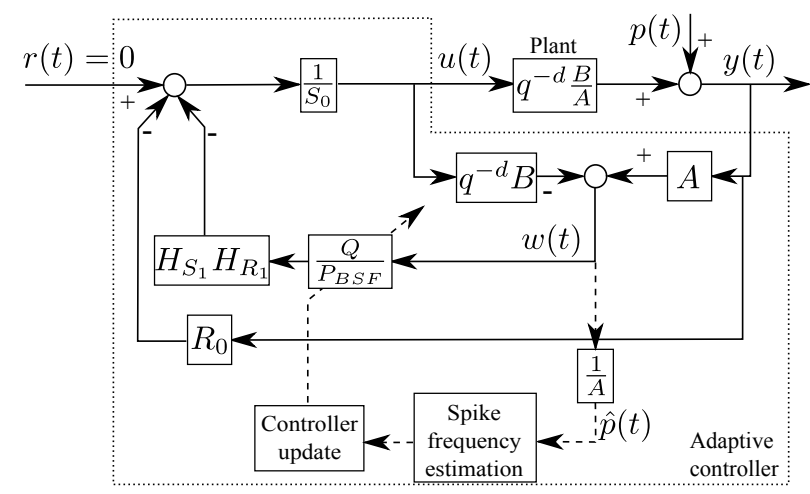

Fig. 2. Youla-Kučera schema for indirect adaptive control.

\section{Frequency Estimation Using Adaptive Notch FILTERS}

In order to use the presented control strategy in the presence on unknown and/or time varying narrow-band disturbances, one needs an estimation in real time of the spikes' frequencies in the spectrum of the disturbance. In the framework of narrow-band disturbance rejection, it is usually supposed that the disturbances are in fact sinusoidal signals with variable frequencies. As specified in the introduction, it is assumed that the number of narrow-band disturbances is known (similar to [1], [4], [27]). A technique based on ANFs will be used to estimate the frequencies of the sinusoidal signals in the disturbance (more details can be found in [16], [23]).

The general form of an ANF is

$$
H_{f}\left(z^{-1}\right)=\frac{A_{f}\left(z^{-1}\right)}{A_{f}\left(\rho z^{-1}\right)},
$$

where the polynomial $A_{f}\left(z^{-1}\right)$ is such that the zeros of the transfer function $H_{f}\left(z^{-1}\right)$ lie on the unit circle. A necessary condition for a monic polynomial to satisfy this property is that its coefficients have a mirror symmetric form

$$
\begin{gathered}
A_{f}\left(z^{-1}\right)=1+a_{1}^{f} z^{-1}+\ldots+a_{n}^{f} z^{-n}+\ldots+ \\
+a_{1}^{f} z^{-2 n+1}+z^{-2 n} .
\end{gathered}
$$

Another requirement is that the poles of the ANF should be on the same radial lines as the zeros but slightly closer to 
the origin of the unit circle. Using filter denominators of the general form $A_{f}\left(\rho z^{-1}\right)$ with $\rho$ a positive real number smaller but close to 1 , the poles have the desired property and are in fact located on a circle of radius $\rho$ ([16]).

The estimation algorithm will be detailed next. It is assumed that the disturbance signal (or a good estimation) is available.

A cascade construction of second order ANF filters is considered. Their number is given by the number of narrowband signals whose frequencies have to be estimated. The main idea behind this algorithm is to consider the signal $\hat{p}(t)$ as having the form

$$
\hat{p}(t)=\sum_{i=1}^{n} c_{i} \sin \left(\omega_{i} \cdot t+\beta_{i}\right)+v(t)
$$

where $v(t)$ is a noise affecting the measurement and $n$ is the number of narrow-band signals with different frequencies.

The ANF cascade form will be given by (this is an equivalent representation of eqs. (30) and (31))

$$
H_{f}\left(z^{-1}\right)=\prod_{i=1}^{n} H_{f}^{i}\left(z^{-1}\right)=\prod_{i=1}^{n} \frac{1+a^{f_{i}} z^{-1}+z^{-2}}{1+\rho a^{f_{i}} z^{-1}+\rho^{2} z^{-2}}
$$

Next, the estimation of one spike's frequency is considered, assuming convergence of the other $n-1$, which can thus by filtered out of the estimated disturbance signal, $\hat{p}(t)$, by applying

$$
\hat{p}^{j}(t)=\prod_{\substack{i=1 \\ i \neq j}}^{n} \frac{1+a^{f_{i}} z^{-1}+z^{-2}}{1+\rho a^{f_{i}} z^{-1}+\rho^{2} z^{-2}} \hat{p}(t) .
$$

The prediction error is obtained from

$$
\epsilon(t)=H_{f}\left(z^{-1}\right) \hat{p}(t)
$$

and can be computed based on one of the $\hat{p}^{j}(t)$ to reduce the computation complexity. Each cell can be adapted independently after prefiltering the signal by the others. Following the Recursive Prediction Error (RPE) technique, the gradient is obtained as

$$
\Psi^{j}(t)=-\frac{\partial \epsilon(t)}{\partial a^{f_{j}}}=\frac{(1-\rho)\left(1-\rho z^{-2}\right)}{1+\rho a^{f_{j}} z^{-1}+\rho^{2} z^{-2}} \hat{p}^{j}(t) .
$$

The parametric adaptation algorithm can be summarized as

$$
\begin{aligned}
\hat{a}^{f_{j}}(t) & =\hat{a}^{f_{j}}(t-1)+F(t-1) \cdot \Psi^{j}(t) \cdot \epsilon(t) \\
F(t) & =\frac{F(t-1)}{\lambda+F(t-1) \Psi^{j}(t)^{2}} .
\end{aligned}
$$

where $\hat{a}^{f_{j}}$ are estimations of the true $a^{f_{j}}$, which are connected to the narrow-band signals' frequencies by $\omega_{f_{j}}=$ $f_{s} \cdot \arccos \left(-\frac{a^{f_{j}}}{2}\right)$, where $f_{s}$ is the sampling frequency.

\section{Stability Analysis of the Adaptive Scheme}

The frequency estimator used (Section V, eqs. (30) through (38)), provided that the number of sinusoidal narrow-bands $(n)$ is known and equal to the number of ANFs (persistence of excitation assumption) and that a decreasing gain adaptation algorithm is used, has the properties, [23],

$$
\begin{aligned}
\lim _{t \rightarrow \infty} \hat{f}_{i}(t) & =f_{i}, \\
\lim _{t \rightarrow \infty}\left(\hat{f}_{i}(t)-\hat{f}_{i}(t-1)\right) & =0, \forall i \in\{1, \ldots n\} .
\end{aligned}
$$

Lets assume that the disturbance can be modelled by ${ }^{7}$ [22]

$$
\begin{aligned}
p(t+1) & =\frac{D\left(\rho q^{-1}\right)}{D\left(q^{-1}\right)} \delta(t+1)=\frac{D(\rho)}{D} \delta(t+1) \\
& =-D^{*} p(t)+D^{*}(\rho) \delta(t)+\delta(t+1),
\end{aligned}
$$

where $D\left(q^{-1}\right)$ has complex zeros on the unit circle, $\rho \in(0,1)$ is an a priori fixed constant, and $\delta(t)$ is a Dirac impulse. The polynomials $D\left(q^{-1}\right)$ and $D\left(\rho q^{-1}\right)$ are given by

$$
\begin{aligned}
D\left(q^{-1}\right) & =\prod_{i=1}^{n}\left(1+\alpha_{i} q^{-1}+q^{-2}\right), \\
D\left(\rho q^{-1}\right) & =\prod_{i=1}^{n}\left(1+\rho \alpha_{i} q^{-1}+\rho^{2} q^{-2}\right),
\end{aligned}
$$

where $n$ is the number of sinusoidal narrow-bands,

$$
\alpha_{i}=-2 \cos \left(\frac{2 \pi f_{i}}{f_{s}}\right) .
$$

Define also the estimate of $D\left(q^{-1}\right)$ obtained from the estimate of $\alpha_{i}$ at time $t$

$$
\begin{aligned}
\hat{D}\left(t, q^{-1}\right) & =\prod_{i=1}^{n}\left(1+\hat{\alpha}_{i}(t) q^{-1}+q^{-2}\right), \\
\hat{\alpha}_{i}(t) & =-2 \cos \left(\frac{2 \pi \hat{f}_{i}(t)}{f_{s}}\right) .
\end{aligned}
$$

For the stability of the full adaptive control scheme, one has the following result:

Theorem 6.1: Consider the indirect adaptive regulation for the plant model (1), given in eqs. (20) through (27), where the estimates of the spikes' frequencies are given by the estimator given in eqs. (30) through (38) using a decreasing gain parametric adaptation algorithm.

Assume that:

H1) The disturbance $p(t)$ is bounded $\forall t$.

$\mathrm{H} 2$ ) The number of sinusoidal narrow-bands is known.

H3) The sinusoidal narrow-bands have unknown but fixed frequencies.

H4) Convergence properties (39) and (40) hold (persistence of excitation and decreasing adaptation gain).

H5) The disturbance model is of the form of eq. (41), where $D\left(q^{-1}\right)$ has complex zeros on the unit circle.

H6) The estimates of $D\left(q^{-1}\right), \hat{D}\left(t, q^{-1}\right)$, are computed from the estimation of the spike frequencies using eqs. (45) and (46).

H7) The BSFs introduced in the output sensitivity function, for a priori fixed constants $\rho_{n}, \rho_{d} \in(0,1)$, are given by

$$
H_{B S F}\left(q^{-1}\right)=\frac{D\left(\rho_{n} q^{-1}\right)}{D\left(\rho_{d} q^{-1}\right)} \text {. }
$$

\footnotetext{
${ }^{7}$ The argument $\left(q^{-1}\right)$ will be dropped in some of the following equations.
} 
H8) $A\left(q^{-1}\right), B\left(q^{-1}\right)$ and the integer time delay $d$ are known. H9) $B\left(q^{-1}\right)$ does not have zeros on the circle of radius $\rho_{n}$.

H10) The assigned closed loop poles $\hat{P}\left(t, q^{-1}\right)$ are inside the unit circle, $\forall t$.

Then:

(1) The sequences $u(t)$ and $y(t)$ are bounded.

(2)

$$
\begin{aligned}
& \lim _{t \rightarrow \infty}[\hat{P}(t) y(t)-\hat{S}(t) \bar{A} p(t)]=0, \\
& \lim _{t \rightarrow \infty}[\hat{P}(t) u(t)+\hat{R}(t) \bar{A} p(t)]=0 .
\end{aligned}
$$

Note that the quantities in the square brackets of eqs. (48) and (49) are zero in the case of known parameters.

The proof can be found in Appendix A.

\section{EXPERIMENTAL RESULTS}

\section{A. An Active Vibration Control System Using an Inertial Actuator}

Figures 3 and 4 represent an AVC system using an inertial actuator for reducing the residual acceleration. The system consists of 5 metallic plates connected by springs. The plates M1 and M3 are equipped with inertial actuators. The one on M1 serves as disturbance generator (inertial actuator 1 in fig. 4), the one on M2 serves for disturbance compensation (inertial actuator 2 in fig. 4). The system is equipped with a measure of the residual acceleration (on plate M3). The path between the disturbance (in this case generated by the inertial actuator on top of the structure), and the residual acceleration is called the primary path. The path between the inertial actuator for compensation and the residual acceleration is called the secondary path $\left(G\left(q^{-1}\right)\right.$ in Section II) and it characterizes the dynamics from the control signal to the residual acceleration measurement (amplifier + actuator + dynamics of the mechanical system). Its orders have been estimated as being $n_{A G}=n_{B G}=14$.

The disturbance is the position of the mobile part of the inertial actuator located on top of the structure (see figs. 3 and 4). The input to the compensator system is the position of the mobile part of the inertial actuator located on the bottom of the structure. A sampling frequency, $f_{s}$, of $800 \mathrm{~Hz}$ has been used.

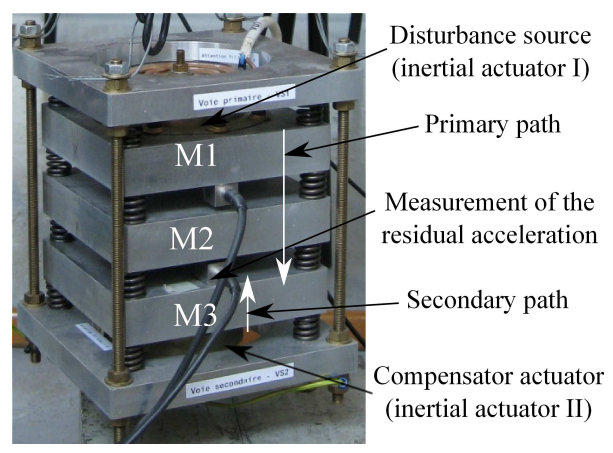

Fig. 3. An AVC system using a feedback compensation - photo.

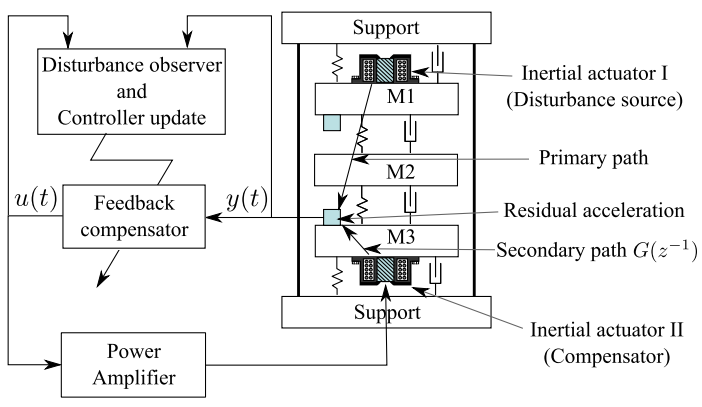

Fig. 4. An AVC system using a feedback compensation - schema.

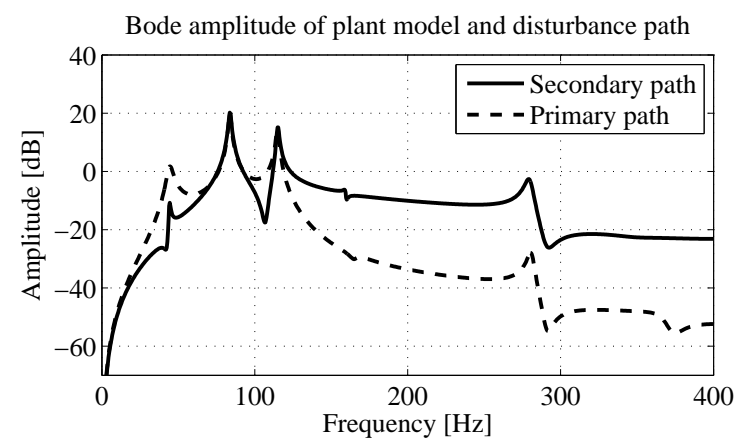

Fig. 5. Secondary and primary paths Bode amplitudes.

\section{B. Attenuation of Multi-sinusoidal Disturbance}

An experimental comparison of the proposed algorithm with the direct adaptive controller of [1] which uses IMP is presented. A multi-sinusoidal signal has been used as disturbance (input to the primary path).

In all of the following experiments, for the design using Youla-Kučera parametrized BSF's, $\zeta_{d}$ has been chosen equal to 0.04 and an attenuation of $60 \mathrm{~dB}$ has been imposed on all of the spikes. The nominal controller's characteristic polynomial, $P_{0}\left(z^{-1}\right)$, contains all the poles of the secondary path model and 15 additional real poles at 0.42 for robustness. Furthermore, the fixed part of the controller's numerator has been chosen as $H_{R_{1}}\left(z^{-1}\right)=1-z^{-2}$, thus opening the loop at $0 \mathrm{~Hz}$ and $400 \mathrm{~Hz}$ (half the sampling frequency), while the fixed part of the controller's denominator was $H_{S_{1}}\left(z^{-1}\right)=1$. The Youla-Kučera IMP design uses the same central controller.

Remark: for the rejection of 3 sine with the adaptive IMP algorithm, 3 pairs of fixed poles with damping 0.2 located within the range of variation of the disturbance spikes' frequencies have been added to the nominal closed loop poles in order to improve robustness outside the attenuation band. For the minimality of the solution, the number of real poles at 0.42 has been reduced to 9 .

Two types of disturbances have been considered: a) with 2 spikes (fig. 6) having a magnitude of 0.1 each and b) with 3 spikes (figs. 7) having a magnitude of 0.04 each, in order to avoid saturation of the control input with the direct adaptive controller of [1] (the system is not well suited for using this method - the actuator does not have enough power). In figs. 6 and 7 , the signals on top represent the effect of the disturbance upon the residual acceleration in open loop operation, the ones in the middle correspond to the residual acceleration in closed 
loop with the proposed BSF algorithm and the ones on the bottom are the residual accelerations obtained with the direct adaptive regulator of [1] using IMP.

Three sequences of multi-sinusoidal disturbances have been applied to the primary path. Their corresponding frequencies are indicated in each of the figures. The first sequence starts at $3 \mathrm{sec}$, and the duration between step changes in frequencies is $10 \mathrm{sec}$.
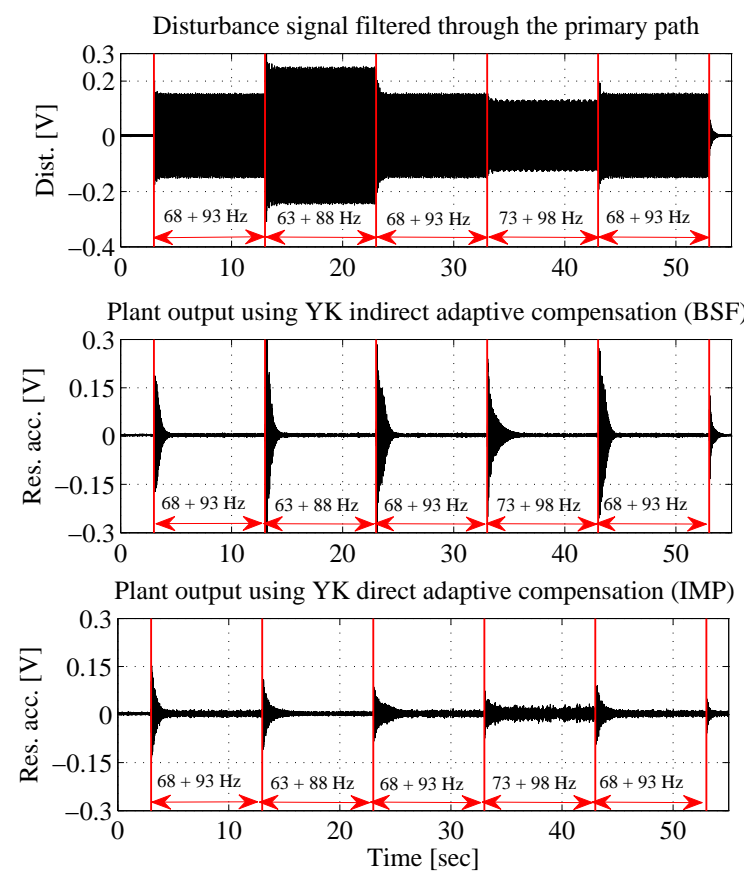

Fig. 6. Performance comparison in the presence of a two sine wave disturbance.

It should be observed that the proposed algorithm has very good stationary disturbance rejection properties but the one of [1] is better with regard to the transient behavior. For 3 sine, the BSF outperforms the IMP controller in term of steady state performance (after the adaptation converges). This can be further confirmed by looking at the power spectral density (PSD) estimates (computed after the adaptation process has converged toward an almost constant controller). In fig. 8, the PSD for 63 and $88 \mathrm{~Hz}$ disturbance are shown. It should be observed that the direct adaptive algorithm of [1] introduces a larger attenuation of the spikes but an amplification of 10 to $20 \mathrm{~dB}$, with respect to the open loop signal, can be observed between 185 and $230 \mathrm{~Hz}$. This influences the global attenuation of the algorithm. A better global attenuation is obtained by the proposed algorithm $(65 \mathrm{~dB})$ in comparison to the direct adaptive algorithm of [1] (54 dB).

Remark: the attenuation of the BSF algorithm at each of the two disturbance frequencies in fig. 8 is of $60 \mathrm{~dB}$ but, due to the small number of points of the window used to compute the PSD, this is not visible in the figure.

\section{CONCluding Remarks}

The technique of BSF for shaping the output sensitivity function [9] is very appropriate for the attenuation of multiple
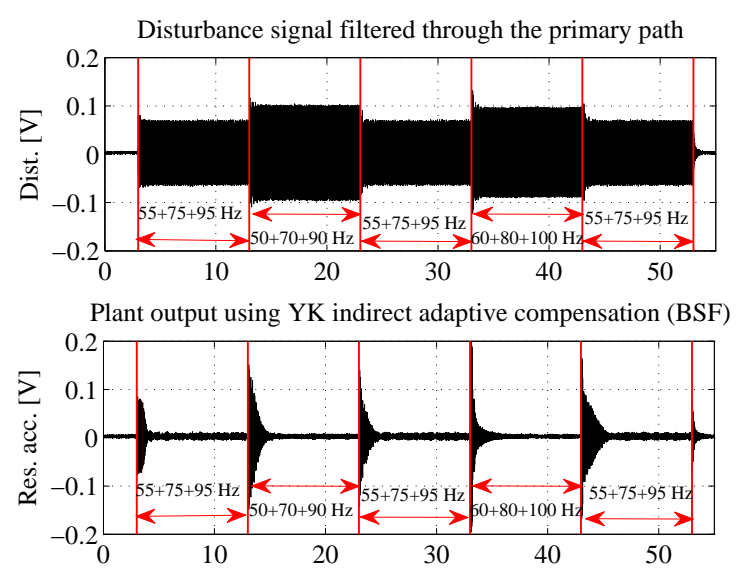

Plant output using YK direct adaptive compensation (IMP)

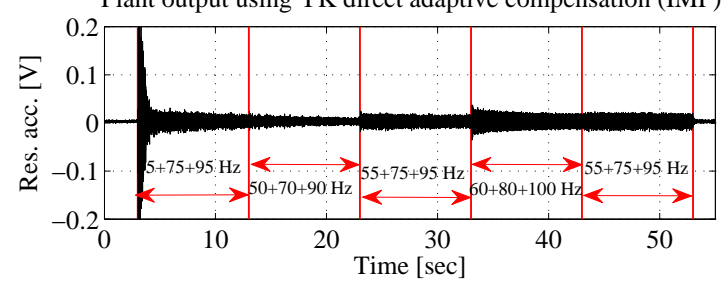

Fig. 7. Performance evaluation in the presence of 3 variable sinusoidal signals.

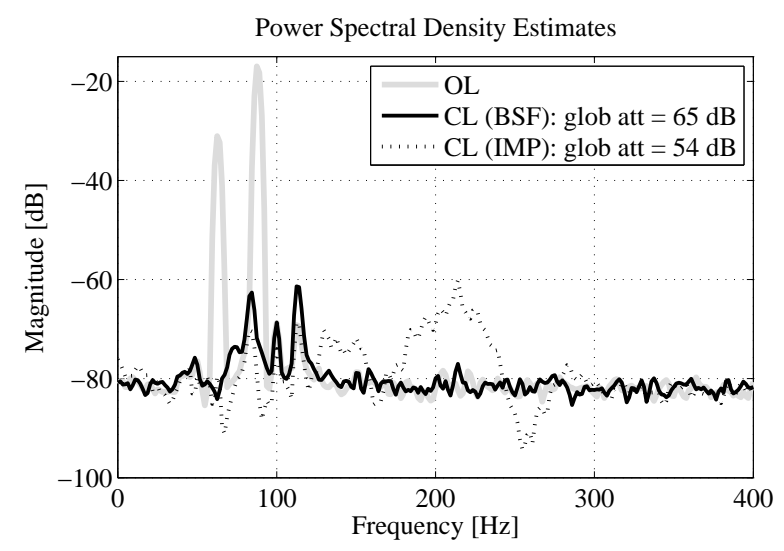

Fig. 8. PSD comparison between the open loop measured disturbance and the residual accelerations obtained with the direct and the indirect compensators (512 points window).

narrow-band disturbances. This design method has been transformed into an adaptive procedure by adding an estimator of the spikes' frequencies characterizing unknown time varying multiple narrow-band disturbances. The experimental results show the potential of this approach for solving practical problems related to attenuation of narrow-band disturbances.

\section{APPENDIX A \\ ProOF OF THEOREM 6.1}

Proof: First, let denote

$$
\varepsilon_{p}(t)=\left(D\left(\rho_{n}\right)-\hat{D}\left(t, \rho_{n}\right)\right) p(t) .
$$


Looking at the properties of the ANF estimator, it can be observed that under hypotheses H1 through H6,

$$
\begin{aligned}
\lim _{t \rightarrow \infty}[D-\hat{D}(t)] & =0, \\
\lim _{t \rightarrow \infty}[\hat{D}(t)-\hat{D}(t-1)] & =0 \\
\hat{D}(t) & \text { has bounded coefficients, }
\end{aligned}
$$

from which it results also that

$$
\begin{aligned}
\lim _{t \rightarrow \infty} & \varepsilon_{p}(t+1)=0 \\
\varepsilon_{p}(t+1) & \text { is bounded. }
\end{aligned}
$$

To prove property (1), one has to write an equation for the evolution of $y(t)$ and $u(t)$. At each time $t$, the following Bezout equation is solved when using BSFs

$$
\hat{P}(t)=P_{0} \hat{D}\left(t, \rho_{d}\right)=A \hat{D}\left(t, \rho_{n}\right) \hat{S}^{\prime}(t)+q^{-d} B \hat{R}(t) .
$$

The notations $A D\left(\rho_{n}\right)=\bar{A}, A \hat{D}\left(t, \rho_{n}\right)=\hat{\bar{A}}(t)$, and $\bar{B}=$ $q^{-d} B$ are introduced to simplify the equations. Considering the augmented model of the secondary path, the system's output becomes

$$
y(t)=\frac{\bar{B}}{\overline{\bar{A}}(t)} \bar{u}(t)+p(t),
$$

where the control signal is given by

$$
\bar{u}(t)=-\frac{\hat{R}(t)}{\hat{S}^{\prime}(t)} y(t),
$$

while the real plant input is $u(t)=\frac{1}{\hat{D}\left(t, \rho_{n}\right)} \bar{u}(t)$. Eq. (57) can be written also as

$$
\begin{aligned}
\hat{\bar{A}}(t) y(t) & =\bar{B} \bar{u}(t)+\hat{\bar{A}}(t) p(t) \pm \bar{A} p(t) \\
\hat{\bar{A}}(t) y(t) & =\bar{B} \bar{u}(t)-A \varepsilon_{p}(t)+\bar{A} p(t)
\end{aligned}
$$

Therefore,

$$
\begin{aligned}
y(t)= & -\sum_{i=1}^{n_{\bar{A}}} \hat{\bar{a}}_{i}(t) y(t-i)+\sum_{i=1}^{n_{\bar{B}}} \bar{b}_{i} \bar{u}(t-i)- \\
& \quad-A \varepsilon_{p}(t)+\bar{A} p(t) \\
= & \hat{\theta}^{T}(t) \phi(t-1)-A \varepsilon_{p}(t)+\bar{A} p(t),
\end{aligned}
$$

where

$$
\begin{aligned}
& \hat{\theta}^{T}(t)=\left[-\hat{\bar{a}}_{1}(t), \ldots-\hat{\bar{a}}_{n_{\bar{A}}}(t), \bar{b}_{1}, \ldots \bar{b}_{n_{\bar{B}}}\right] \\
& \phi^{T}(t)=\left[y(t), \ldots y\left(t-n_{\bar{A}}+1\right),\right. \\
&\left.\bar{u}(t), \ldots \bar{u}\left(t-n_{\bar{B}}+1\right)\right] .
\end{aligned}
$$

Similarly, from (58) and (62),

$$
\begin{aligned}
\hat{S}^{\prime}(t) u(t)= & -\hat{R}^{*}(t) y(t-1)-\hat{r}_{0}(t)\left[\hat{\theta}^{T} \phi(t-1)\right]+ \\
& +\hat{r}_{0}(t) A \varepsilon_{p}(t)-\hat{r}_{0}(t) \bar{A} p(t) .
\end{aligned}
$$

Let denote

$$
\begin{aligned}
& \hat{\bar{r}}_{i}(t)=\hat{r}_{i}(t)-\hat{r}_{0}(t) \hat{\bar{a}}_{i}(t), \forall i \in\left\{1, \ldots n_{\bar{A}}\right\} \\
& \hat{\bar{s}}_{i}(t)=\hat{s}_{i}^{\prime}(t)+\hat{r}_{0}(t) \bar{b}_{i}, \quad \forall i \in\left\{1, \ldots n_{\bar{B}}\right\} .
\end{aligned}
$$

With these notations, (65) becomes

$$
\begin{aligned}
u(t)= & -\sum_{i=1}^{n_{\bar{B}}} \hat{\bar{s}}_{i}(t) u(t-i)-\sum_{i=1}^{n_{\bar{A}}} \hat{\bar{r}}_{i}(t) y(t-i)+ \\
& +\hat{r}_{0}(t) A \varepsilon_{p}(t)-\hat{r}_{0}(t) \bar{A} p(t) .
\end{aligned}
$$

Combining now (61) and (68) as in (12.38) of [29], one obtains a state space equation for $\phi(t)$

$$
\phi(t)=L(t) \phi(t-1)+z(t),
$$

where the $\mathbb{R}^{\left(n_{\bar{A}}+n_{\bar{B}}\right) \times\left(n_{\bar{A}}+n_{B}\right)}$ matrix $L(t)$ is given by

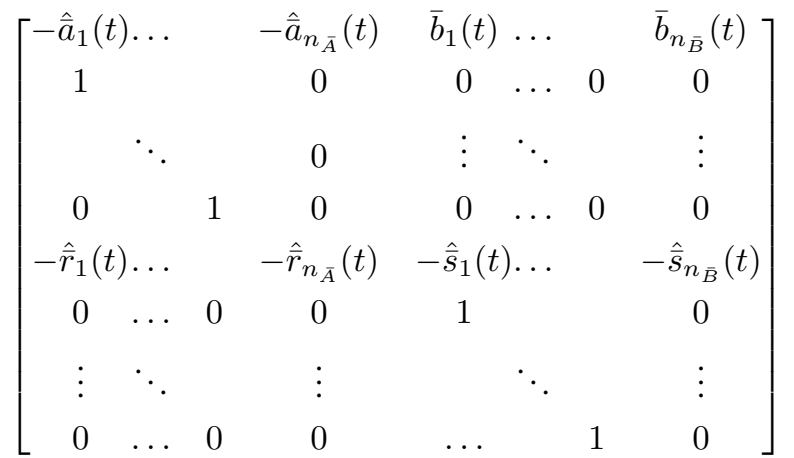

and

$$
z^{T}(t)=\left[\begin{array}{ll}
z_{y}^{T}(t) & z_{u}^{T}(t)
\end{array}\right]
$$

is a vector in $\mathbb{R}^{n_{\bar{A}}+n_{\bar{B}}}$, where

$$
\begin{aligned}
z_{y}^{T}(t) & =\left[\begin{array}{lll}
\bar{A} p(t)-A \varepsilon_{p}(t) & 0 & \ldots
\end{array}\right] \in \mathbb{R}^{n_{\bar{A}}}, \\
z_{u}^{T}(t) & =\left[\begin{array}{llll}
\hat{r}_{0}(t) A \varepsilon_{p}(t)-\hat{r}_{0}(t) \bar{A} p(t) & 0 & \ldots
\end{array}\right] \in \mathbb{R}^{n_{\bar{B}}} .
\end{aligned}
$$

To prove the boundedness of $\phi(t)$, Lemma 12.1 of [29] will be used. The first condition of the lemma is evident since the coefficients of $L$ are finite for all $t$. Taking into account the fact that the eigenvalues of the matrix $L(t),(70)$, are given by $\lambda^{n_{\bar{A}}+n_{\bar{B}}-1} \hat{P}(t, \lambda)=0$ (see [29]) and knowing that the poles of the closed loop are chosen inside the unit circle, the eigenvalues of the matrix $L(t)$ are also inside the unit circle, hence the second condition is also satisfied. The last condition is satisfied under hypothesis $\mathrm{H} 4$ which will imply

$$
\lim _{t \rightarrow \infty}[L(t)-L(t-1)]=0 .
$$

It results that $\exists t^{*}$, s.t. $\forall t \geq t^{*}$ :

$$
\begin{aligned}
& \|\phi(t+1)\|^{2} \leq \\
& \quad \leq C_{1}^{\prime}+C_{2}^{\prime} \max _{0 \leq \tau \leq t}\left[\left(1-\hat{r}_{0}(\tau)^{2}\right)\left(\bar{A} p(\tau)-A \varepsilon_{p}(\tau)\right)^{2}\right] \\
& \quad \leq C_{1}+C_{2} \max _{0 \leq \tau \leq t} \varepsilon_{p}(\tau)^{2} .
\end{aligned}
$$

since $p(t)$ is bounded. Therefore, $\forall t \geq t^{*}, \phi(t+1)$ is bounded since $\varepsilon_{p}(t)$ is bounded and since $u(t)=\frac{1}{\hat{D}\left(t, \rho_{n}\right)} \bar{u}(t), u(t)$ and $y(t)$ are also bounded.

To prove property (2), one has to write the expressions of the quantities mentioned in eqs. (48) and (49). We introduce the notations:

$$
\begin{array}{ll}
\Delta_{11}(t)=\hat{\bar{A}}(t) \hat{S}(t)-\hat{S}(t) \hat{\bar{A}}(t), & \Delta_{12}(t)=\hat{S}(t) \bar{B}-\bar{B} \hat{S}(t), \\
\Delta_{21}(t)=\hat{R}(t) \hat{\bar{A}}(t)-\hat{\bar{A}}(t) \hat{R}(t), \quad \Delta_{22}(t)=\bar{B} \hat{R}(t)-\hat{R}(t) \bar{B} .
\end{array}
$$


From (56) and (60),

$$
\begin{aligned}
\hat{P}(t) y(t)=\hat{S}(t) & \left(\bar{B} \bar{u}(t)-A \varepsilon_{p}(t)+\bar{A} p(t)\right)+ \\
& +\bar{B} \hat{R}(t) y(t)+\Delta_{11}(t) y(t)
\end{aligned}
$$

and taking into account (58)

$$
\bar{B} \hat{R}(t) y(t)+\bar{B} \hat{S}(t) \bar{u}(t)=0
$$

one obtains

$$
\begin{aligned}
\hat{P}(t) y(t)=\hat{S}(t) & \bar{A} p(t)-\hat{S}(t) A \varepsilon_{p}(t)+ \\
& +\Delta_{11}(t) y(t)+\Delta_{12}(t) \bar{u}(t) .
\end{aligned}
$$

Similarly,

$$
\begin{gathered}
\hat{P}(t) \bar{u}(t)=\hat{\bar{A}}(t) \hat{S}(t) \bar{u}(t)+\hat{R}(t) \hat{\bar{A}}(t) y(t)-\hat{R}(t) \bar{A} p(t)+ \\
\quad+\hat{R}(t) A \varepsilon_{p}(t)+\Delta_{22}(t) \bar{u}(t) \\
=-\hat{R}(t) \bar{A} p(t)+\hat{R}(t) A \varepsilon_{p}(t)+ \\
\quad+\Delta_{22}(t) \bar{u}(t)+\Delta_{21}(t) y(t) .
\end{gathered}
$$

Which lead to eqs. (48) and (49). Taking into account that the various $\Delta_{i j}$ as well as $\varepsilon_{p}(t)$ go to zero, the second property of Theorem 6.1 is also true.

\section{REFERENCES}

[1] I. Landau, M. Alma, J. Martinez, and G. Buche, "Adaptive suppression of multiple time-varying unknown vibrations using an inertial actuator," Control Systems Technology, IEEE Transactions on, vol. 19, no. 6, pp. $1327-1338$, nov. 2011

[2] B. Francis and W. Wonham, "The internal model principle of control theory," Automatica, vol. 12, no. 5, pp. 457 - 465, 1976.

[3] G. Bengtsson, "Output regulation and internal models - a frequency domain approach," Automatica, vol. 13, no. 4, pp. 333 - 345, 1977.

[4] I. Landau, A. Constantinescu, and D. Rey, "Adaptive narrow band disturbance rejection applied to an active suspension - an internal model principle approach," Automatica, vol. 41, no. 4, pp. 563-574, 2005.

[5] M. Ficocelli and F. Ben Amara, "Adaptive regulation of mimo linear systems against unknown sinusoidal exogenous inputs," International Journal of Adaptive Control and Signal Processing, vol. 23, no. 6, pp. 581-603, 2009. [Online]. Available: http://dx.doi.org/10.1002/acs.1072

[6] S. Valentinotti, "Adaptive rejection of unstable disturbances: Application to a fed-batch fermentation," Thèse de doctorat, École Polytechnique Fédérale de Lausanne, April 2001.

[7] T.-B. Airimitoaie, "Robust design and tuning of active vibration control systems," Ph.D. dissertation, University of Grenoble, France, 2012.

[8] I. Landau and G. Zito, Digital Control Systems - Design, Identification and Implementation. London: Springer, 2005.

[9] H. Prochzka and I. D. Landau, "Pole placement with sensitivity function shaping using 2nd order digital notch filters," Automatica, vol. 39, no. 6, pp. $1103-1107,2003$.

[10] Y. Tsypkin, "Stochastic discrete systems with internal models," Journal of Automation and Information Sciences, vol. 29, no. 4\&5, pp. 156-161, 1997.

[11] R. A. de Callafon and C. E. Kinney, "Robust estimation and adaptive controller tuning for variance minimization in servo systems," Journal of Advanced Mechanical Design, Systems, and Manufacturing, vol. 4 , no. 1 , pp. $130-142,2010$.

[12] T. T. Tay, I. M. Y. Mareels, and J. B. Moore, High Performance Control. Birkh auser Boston, 1997.

[13] T.-B. Airimitoaie, I. Landau, L. Dugard, and D. Popescu, "Identification of mechanical structures in the presence of narrow band disturbances application to an active suspension," in Control Automation (MED), 2011 19th Mediterranean Conference on, june 2011, pp. $904-909$.

[14] P. Tichavský and A. Nehorai, "Comparative study of four adaptive frequency trackers," IEEE Trans. Autom. Control, vol. 45, no. 6, pp. 1473 - 1484, 1997.

[15] D. Rao and S.-Y. Kung, "Adaptive notch filtering for the retrieval of sinusoids in noise," Acoustics, Speech and Signal Processing, IEEE Transactions on, vol. 32, no. 4, pp. 791 - 802, aug 1984.
[16] A. Nehorai, "A minimal parameter adaptive notch filter with constrained poles and zeros," IEEE Trans. Acoust., Speech, Signal Processing, vol. ASSP-33, pp. 983-996, 1985.

[17] P. A. Regalia, "An improved lattice-based adaptive iir notch filter," Signal Processing, IEEE Transactions on, vol. 9, no. 9, pp. 2124 - 2128, september 1991.

[18] B.-S. Chen, T.-Y. Yang, and B.-H. Lin, "Adaptive notch filter by direct frequency estimation,” Signal Processing, vol. 27, no. 2, pp. $161-176$, 1992.

[19] G. Li, "A stable and efficient adaptive notch filter for direct frequency estimation," Signal Processing, IEEE Transactions on, vol. 45, no. 8 , pp. $2001-2009$, aug 1997.

[20] L. Hsu, R. Ortega, and G. Damm, "A globally convergent frequency estimator," IEEE Trans. Autom. Control, vol. 4, no. 4, pp. 698-713, 1999.

[21] G. Obregon-Pulido, B. Castillo-Toledo, and A. Loukianov, "A globally convergent estimator for n-frequencies," IEEE Trans. Autom. Control, vol. 47, no. 5, pp. 857-863, 2002.

[22] P. Stoica and A. Nehorai, "Performance analysis of an adaptive notch filter with constrained poles and zeros," IEEE Trans. Acoust., Speech, Signal Processing, vol. 36, no. 6, pp. $911-919,1988$.

[23] N. M'Sirdi, H. Tjokronegoro, and I. Landau, "An rml algorithm for retrieval of sinusoids with cascaded notch filters," in Acoustics, Speech, and Signal Processing, 1988. ICASSP-88., 1988 International Conference on, apr 1988, pp. $2484-2487$ vol.4.

[24] M. Bodson, "Rejection of periodic distrubances of unknown and timevarying frequency," Int. J. of Adapt. Contr. and Sign. Proc., vol. 19, pp. $67-88,2005$.

[25] Y. Huang and W. Messner, "A novel disturbance observer design for magnetic hard drive servo system with a rotary actuator," Magnetics, IEEE Transactions on, vol. 34, no. 4, pp. 1892 -1894, jul 1998.

[26] Q. Zheng and M. Tomizuka, "A disturbance observer approach to detecting and rejecting narrow-band disturbances in hard disk drives," in Advanced Motion Control, 2008. AMC '08. 10th IEEE International Workshop on, March 2008, pp. $254-259$.

[27] X. Chen and M. Tomizuka, "A minimum parameter adaptive approach for rejecting multiple narrow-band disturbances with application to hard disk drives," Control Systems Technology, IEEE Transactions on, vol. 20, no. 2, pp. $408-415$, march 2012.

[28] M. Bodson and S. Douglas, "Adaptive algorithms for the rejection of sinusosidal disturbances with unknown frequency," Automatica, vol. 33, pp. 2213-2221, 1997.

[29] I. D. Landau, R. Lozano, M. M'Saad, and A. Karimi, Adaptive control, 2nd ed. London: Springer, 2011. 